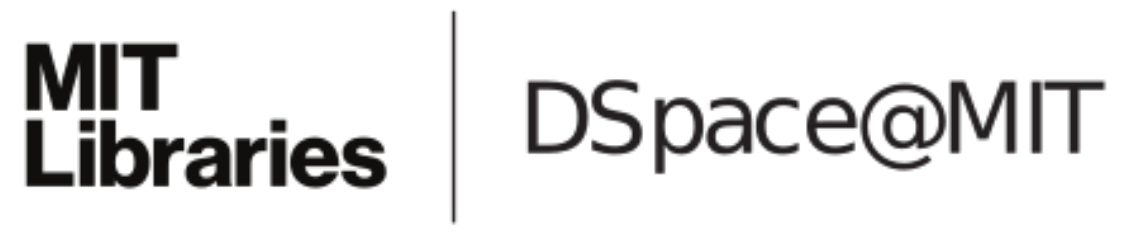

\author{
MIT Open Access Articles
}

Phase behavior of symmetric disk-coil molecules

The MIT Faculty has made this article openly available. Please share how this access benefits you. Your story matters.

Citation: Kim, Yongjoo, and Alfredo Alexander-Katz. Phase Behavior of Symmetric Disk-coil Molecules. The Journal of Chemical Physics 132, no. 17 (2010): 174901.@ 2010 American Institute of Physics.

As Published: http://dx.doi.org/10.1063/1.3407660

Publisher: American Institute of Physics

Persistent URL: http://hdl.handle.net/1721.1/79419

Version: Final published version: final published article, as it appeared in a journal, conference proceedings, or other formally published context

Terms of Use: Article is made available in accordance with the publisher's policy and may be subject to US copyright law. Please refer to the publisher's site for terms of use. 


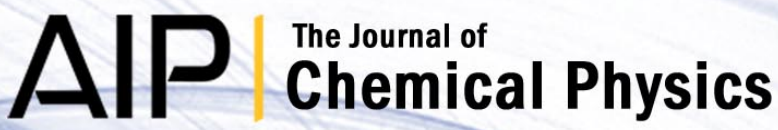

\section{Phase behavior of symmetric disk-coil macromolecules with stacking interactions}

YongJoo Kim and Alfredo Alexander-Katz

Citation: J. Chem. Phys. 135, 024902 (2011); doi: 10.1063/1.3607985

View online: http://dx.doi.org/10.1063/1.3607985

View Table of Contents: http://jcp.aip.org/resource/1/JCPSA6/v135/i2

Published by the AIP Publishing LLC.

\section{Additional information on J. Chem. Phys.}

Journal Homepage: http://jcp.aip.org/

Journal Information: http://jcp.aip.org/about/about_the_journal

Top downloads: http://jcp.aip.org/features/most_downloaded

Information for Authors: http://jcp.aip.org/authors

\section{ADVERTISEMENT}

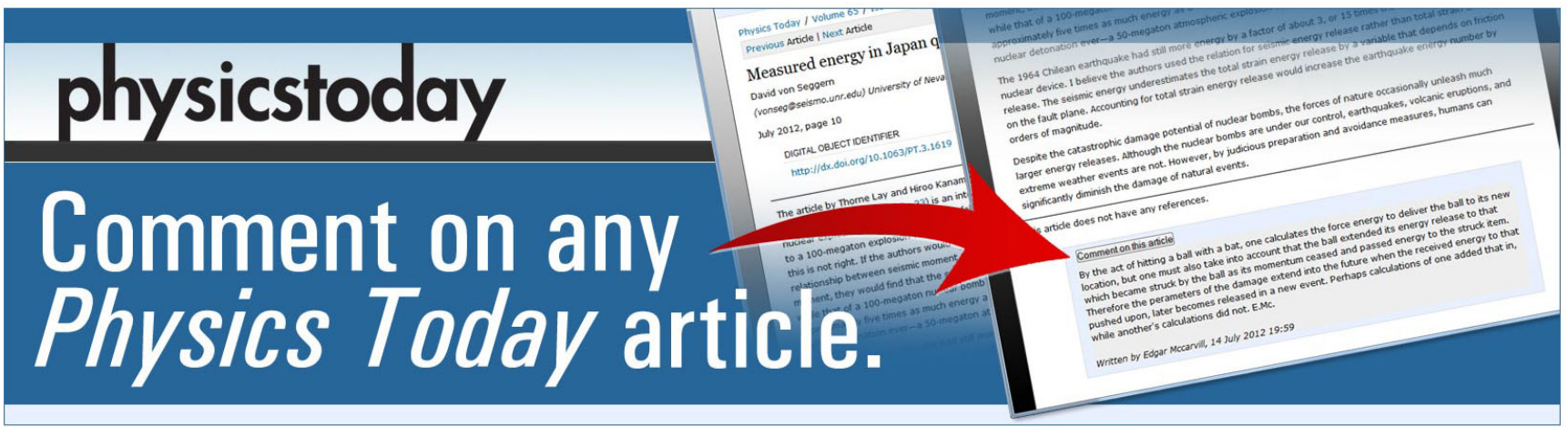




\title{
Phase behavior of symmetric disk-coil macromolecules with stacking interactions
}

\author{
YongJoo Kim and Alfredo Alexander-Katz ${ }^{\mathrm{a})}$ \\ Department of Materials Science and Engineering, MIT, Cambridge, Massachusetts 02139, USA
}

(Received 17 February 2011; accepted 13 June 2011; published online 12 July 2011)

\begin{abstract}
We investigate using Monte Carlo simulations in the NPT ensemble the self-assembly of disk-coil macromolecules with stacking interactions. The disk-coil molecules are composed of a planar disk that is covalently bonded to a single coil. In addition to commonly used amphiphilic interactions between the disk and coil portion of the molecules, we employ an attractive interaction between central monomers of the disks, which mimics stacking interactions. This additional force induces a preferential axial packing. The phase diagram of this system is complex and depends crucially on the stacking interactions. In particular, we find a variety of new phases that include for this system an ordered lamellar, ordered perforated lamellar, cylinder and ordered cylinder phases in addition to the disordered, lamellar, perforated lamellar, and crystal phases observed previously [Y. Kim and A. Alexander-Katz, J. Chem. Phys. 132, 174901 (2010)]. The ordered phases show strong tendency of parallel packing of disks. Among them, the ordered cylinder phase exhibits super-aligned structures which could have uses in many organic optoelectronic applications. (C) 2011 American Institute of Physics. [doi:10.1063/1.3607985]
\end{abstract}

\section{INTRODUCTION}

Discotic liquid crystals (DLCs) have been widely studied because of their promising semiconducting properties. ${ }^{1-3}$ Stacked planar molecules driven by $\pi-\pi$ orbital overlapping allows for high electron and charge carrier mobility. For example, hexagonally closed packed columnar stacks can yield high charge carrier mobility along the axis of the columns. ${ }^{1,4}$ However, the processing of the extended pi conjugated systems is essentially impossible due to the strong interactions that frustrate their assembly. In order to help in the processing of these molecules and, in fact, facilitate their assembly into columns, the planar cores are functionalized with coils of the alkyl type. The presence of these tails brings an extra ingredient into the assembly which causes the system to phase separate in a similar fashion as block copolymer dendrons. ${ }^{5-7}$

Synthetic discotics have typically a symmetric corona of coils, with several chains emanating from the periphery of the planar part. ${ }^{4,8,9}$ However, there have been realizations in which the functionalization consists of a single coil. ${ }^{10,11}$ Furthermore, in nature one finds that chlorophyll contains also only one "long" tail, and has been observed to form ordered phases that are not hexagonally close packed columns, but "undulating lamellae"12,13 or "multi-layered tubules"14 structures. The chlorophyll assemblies behave as photosynthetic antennas, so-called chlorosomes, that capture photons in extremely low intensity environments and have been shown to strongly enhance the adsorption of photons in dye-sensithyzed solar cells. ${ }^{15}$ This opens the question as to what parameters determine the phase behavior of disk-coil macromolecules. We have recently addressed this problem and showed that lamellar (L), perforated lamellar (PL), and crystal phases are possible in the

\footnotetext{
a)Electronic mail: aalexand@ mit.edu
}

simplest amphiphilic system with a uniform disk. ${ }^{16}$ The appearance of a perforated phases is determined by the entropic contribution of the coils measured by the parameter $\lambda$ and has been observed also in rod-coil systems. ${ }^{17}$ In the crystal phase, the disks are closed packed within a lamellae and the director field vectors are correlated by $60^{\circ}$ due to the onedimensional confinement of disks. However, columnar stacking, which is desired in many applications such as organic transistors and photoactive devices, does not appear in this system. The model we studied previously is the simplest realization of such a disk-coil macromolecule, and a more realistic description would include further interactions that somehow prefer face-to-face stacking. To understand this, one can think of having a corona of very small coils and one long taillike in block copolymer cylinders. The central part of the disk would then prefer to stack strongly with the other central disk monomers. Here, we consider such a model in which we include additional center-center interactions and find a wealth of new phases, including cylindrical ordered and liquid phases.

We study the disk-coil system using a NPT ensemble with the variable cell-shape method. ${ }^{18,19}$ Theoretical modeling of similar systems has been useful to identify the properties that determine the phases that are possible and also the temperatures at which these molecules undergo the order-disorder transition. The pioneering work of Frenkel and Veerman used a cut-sphere model ${ }^{20}$ and showed that the hexatic columnar arrangement is possible, but needed unphysical (high) pressures to obtain it, which meant that columnar structures are unstable in atmospheric or low pressure condition unless enthalpic contributions are added. On the other hand, the Gay-Berne potential ${ }^{21,22}$ that has been widely used to study discotics did exhibit the columnar phases at moderate pressures, but the ellipsoidal form of the potential does not exactly represent the planar molecule nature of DLCs and 
intercalation is observed, which is not observed experimentally. Refinements to both of these potentials to describe more realistic molecules have been done. In particular, researchers have added dipole-dipole interactions to address chemical modifications in the core that produce redistribution of the charges. The dipoles have been placed at the center of the molecules and the periphery. The qualitative features of the phase diagrams have remained the same, but the quantitative aspects have changed. ${ }^{23}$ As mentioned above, this is not the case in the disk-coil system as we observe new phases appear as well as ordering of only the disk portion of the macromolecules preceding the fully crystallized phase. In particular, we find ordered lamellar, ordered perforated lamellar, cylinder, and ordered cylinder phases as a function of attractive force of central monomers of disks. The ordered cylinder phase shows very high degree of parallel packing of disks compared to other phases. Presumably, it can be possible to design synthetic molecules with the architectures we present here and observe the predicted phases.

The article is organized as follows: Section II describes the molecular model and computational details. We present our results in Sec. III which is divided into three subsections. In Sec. III A, we explain the additional ordered phases due to the added stacking interactions between disks. In Sec. III B, we construct phase diagrams and explain some trends appearing on these phase diagrams. We analyze additional ordered phases to study parallel packing of disks in Sec. III C. Finally, in Sec. IV, we present our concluding remarks.

\section{MOLECULAR MODEL AND COMPUTATIONAL DETAILS}

We study the self-assembly of nearly symmetric diskcoil molecules with additional interaction between the central monomers of the disks. The disk-coil model we use is explained in detail in Ref. 16, and here we briefly describe it. The disk portion of the molecule consists of seven monomers which are numbered by 1-7 in Fig. 1. Nearestneighbor monomers are connected by stiff springs, and additional springs are connected between the following pairs of monomers: $(2,5),(3,6)$, and $(4,7)$ to prevent bending or folding of disk. The coil part of the molecule consists of five monomers (numbered 8-12), and every monomer is connected by a spring to its nearest-neighbor monomer. The potential energy of these springs is harmonic with an equilibrium distance of $r_{e q}=2^{1 / 6} \sigma$, where $\sigma$ is the Lennard-Jones parameter. For the springs between the pairs of monomers $(2,5),(3,6)$, and $(4,7)$, we use an equilibrium distance of $2 r_{e q}$.

The intermolecular potential energy of disk-coil molecules is described by a typical Lennard-Jones potential given by Eq. (1),

$$
U_{\text {interatomic }}=\sum_{j>i}^{12 \times N} 4 \epsilon \chi_{i j}\left[\left(\frac{\sigma}{r}\right)^{12}-\left(\frac{\sigma}{r}\right)^{6}\right],
$$

where $i$ and $j$ represent the monomer index and the total number of monomers is $12 \times N$, where $N$ is the number of diskcoil molecules. Different parts of the molecule have different potential well depths which is described by $\epsilon \times \chi_{i j}$. The dif-

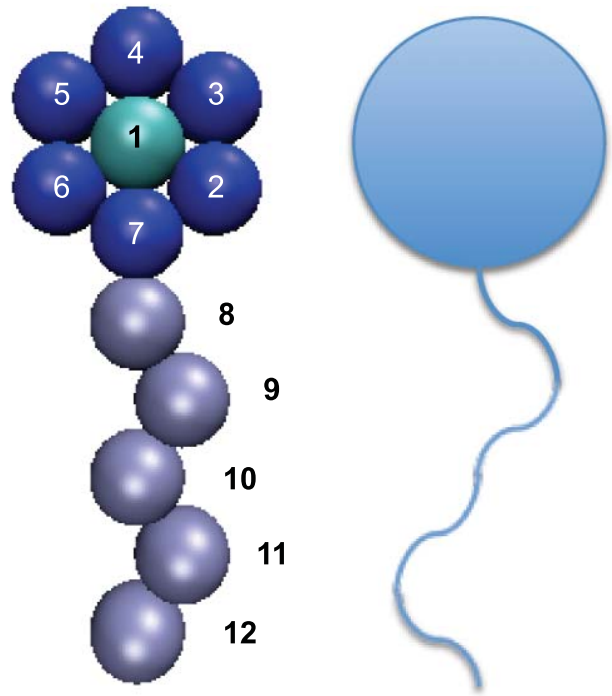

FIG. 1. Model for a disk-coil molecule. Blue monomers (1-7) represent the disk portion and silver monomers (8-12) represent the coil portion. The central monomer (1) of the disk portion is highlighted in green.

ferent $\chi_{i j}$ values as a function of index $i$ and $j$ are listed in Table I.

For monomers in the disk periphery interacting with other disk beads, we put $\chi=1$. Similarly, between two monomers from the coil portion, $\chi$ is set to $\lambda$. In other words, any two monomers of the same type (disk-disk or coil-coil) should be attracted to each other with a potential well depth of $\epsilon$ and $\epsilon \lambda$, respectively. To allow steric repulsion between disk and coil portions of the molecules, but retain the amphiphilic nature of molecules, we employ an additional Weeks-Chandler-Andersen potential between the coil and disk portions. ${ }^{24}$ Finally, when considering the interactions between central monomers in the disks (monomer 1), we set $\chi$ equal to $\mu$ which produces an additional attractive potential that prefers parallel stacking of disks. When $\mu=1$, we recover the uniform disk result. ${ }^{16}$ This interaction is new compared to our previous work and drives the system into novel phases, as shown below.

The dimensionless parameter $\lambda$ represents the relative attractive force between coil portions relative to the disk portions (i.e., the coil-coil potential well depth is $\epsilon \lambda$ and the disk-disk potential well depth is $\epsilon$ ). Thus, by adjusting $\lambda$, we control the role of the coil to be enthalpic (high $\lambda$ value) or entropic (low $\lambda$ value). Also, by adjusting the additional attractive potential $\mu$ between central monomers of disks we control the relative role of disk stacking with respect to the tails.

TABLE I. $\chi_{i j}$ between different type of monomers.

\begin{tabular}{lccccc}
\hline \hline Monomer $i$ & Monomer $j$ & $\chi_{i j}$ & Monomer $i$ & Monomer $j$ & $\chi_{i j}$ \\
\hline $1-7$ & $2-7$ & 1 & $8-12$ & $8-12$ & $\lambda$ \\
$1-7$ & $8-12$ & 0 & 1 & 1 & $\mu$ \\
\hline \hline
\end{tabular}


Reduced variables are used in this simulation; temperature $T^{*}=T \times\left(k_{B} / \epsilon\right)$, distance $r^{*}=r \times(1 / \sigma)$, volume $V^{*}=V \times\left(1 / \sigma^{3}\right)$, pressure $P^{*}=P \times\left(\sigma^{3} / \epsilon\right)$, enthalpy $H^{*}=H \times(1 / \epsilon)$, volume density $\rho^{*}=\rho$, and spring constant $k^{*}=k \times\left(\sigma^{2} / \epsilon\right)$. The simulations are performed for a set of reduced temperatures $T^{*}$ that range from 1.2 to 1.6 , and we explicitly study different $\lambda$ values ranging from 0.1 to 1.0. We also change the $\mu$ value from 1.0 to 6.0. We limit the value of $\mu$ to 6 because when $\mu$ exceed 6.0 the system freezes at metastable states due to strong attractions between center monomers. Also, to prevent the simulation cell to expand infinitely in the high $T^{*}$ phases, we imposed a small but finite value of hydrostatic pressure $P^{*}=0.1$. We set the spring constant of the harmonic potentials $k^{*}$ to 2000 and the extra bond spring constant $2 k^{*}$ to 4000 . Further computational details can be found in Ref. 16.

\section{RESULTS AND DISCUSSION}

\section{A. Ordered phases}

At $\mu=1.0$, disk-coil molecules without additional central monomer interaction self-assemble into four different phases from random initial configurations depending on $T^{*}$ and $\lambda$ : disordered (D), lamellar (L), perforated lamellar (PL), and crystal phase (C). ${ }^{16}$ Because of the enthalpic contribution of disks, disk-coil molecules form a crystal phase at low $T^{*}$ regardless of the role of the coil $(\lambda)$. However, we expect a different temperature of the crystallization of disks when $\mu$ is higher than 1 because of the additional central monomer interaction. We also expect that ordering in the crystal phase at higher $\mu$ to be somehow different to the case of $\mu=1$ because attractive forces between central monomers of disks will cause preferred face-to-face packing of disks. From now on, we name crystallized phases at higher $\mu$ as "ordered phases."

From our simulation results, we obtain four additional phases compared to the $\mu=1$ case: ordered lamellar (L_O), ordered perforated lamellar ( $\left.\mathrm{PL} \_\mathrm{O}\right)$, cylinder $(\mathrm{Cy})$, and ordered cylinder (Cy_O) phases. Typical side views of these phases are shown in Fig. 2. The L_O and PL_O have similar side views, so it is hard to distinguish them. Similarly, it is hard to distinguish between $\mathrm{Cy}$ and Cy_O. To show how disks are arranged in the disk-rich region of these phases, we (a)

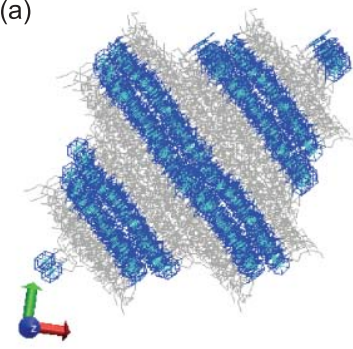

(b)

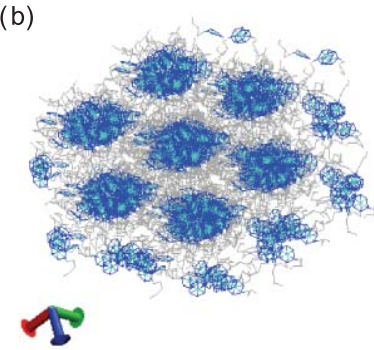

FIG. 2. Side view snapshots of the different self-assembled phases of diskcoil molecules. Blue regions represent the disk portion, and grey regions represent the coil portion. (a) Ordered lamellar and ordered perforated lamellar phases. (b) Cylinder and ordered cylinder phases.

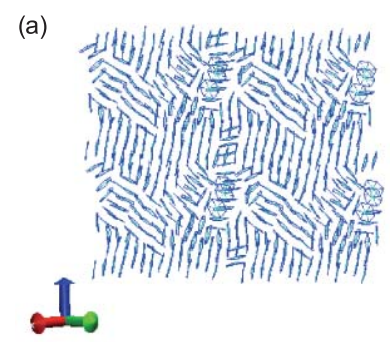

(c)

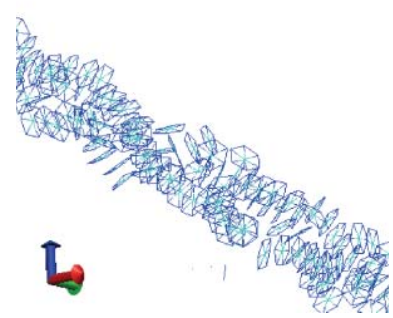

(b)

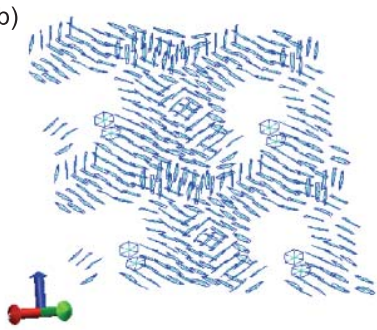

(d)

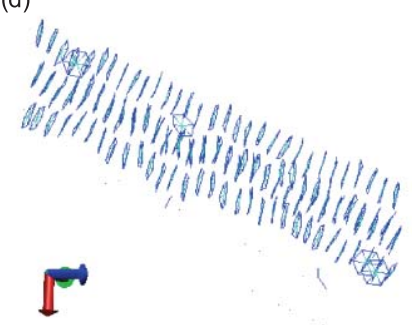

FIG. 3. Top views of one layer of (a) ordered lamellar phase and (b) ordered perforated lamellar phase. One cylinder views of (c) cylinder phase and (d) ordered cylinder phase. We removed coil portion of disk-coil molecule to see arrangement of disks more clearly.

display top views of L_O and PL_O phases, and one cylinder views of $\mathrm{Cy}$ and Cy_O phases without showing the coils in Fig. 3. In the ordered lamellar phase, 4-6 parallel stacked disk rods are closed packed in random directions in lamellar phase-like grains of parallel stacked disk layers. The ordered perforated lamellar phase is somewhat similar to the arrangement of disks in the ordered lamellar phase except that the disk-rich region is perforated. Notice also the faceting of the pores. The cylinder phase is a hexagonally closed packed disk-rich phase similar to that appearing in block copolymers. The ordered cylinder phase has super-parallel stacked disks within the cylinder phase as can be seen in Fig. 3(d).

Like the $\mathrm{L} \rightarrow \mathrm{C}$ or $\mathrm{PL} \rightarrow \mathrm{C}$ phase transitions from earlier paper, transitions to ordered phases are also first order. Figure 4 represents the enthalpy $H^{*}$ and density $\rho^{*}$ versus temperature $T^{*}$ in some $\mu$ and $\lambda$ conditions near ordering transition points. Clear discontinuities of $H^{*}$ and $\rho^{*}$ by decreasing $T^{*}$ near all ordering transition points tell that $\mathrm{L}$ $\rightarrow$ L_O, PL $\rightarrow$ PL_O, and $\mathrm{Cy} \rightarrow$ Cy_O transitions are first order phase transitions. Based on these, we construct phase diagrams as a function of $\lambda$ and $T^{*}$ for six different $\mu$ values in Fig. 5. We explain the details about these phase diagrams in the next subsection.

\section{B. Phase diagram}

Figure 5 shows phase diagrams in $T^{*}$ and $\lambda$ plane for six different $\mu$ values. At intermediate temperature, where $T^{*}$ is not low enough to crystallize disks at $\mu=1.0$, (enthalpic) coils (high $\lambda$ ) prefer to phase separate in a similar fashion to lamellar phase of block copolymers. However, in the case of low $\lambda$, where the entropy of the coils dominates, the system opens up holes in the disk rich region to increase entropy of the coils by increasing their overall available volume. The increase in entropy from opening holes in the disk-rich bilayer compensates and even exceeds the enthalpic loss of disks 
(a) $\quad \mu=4.0 \quad \lambda=1.0$

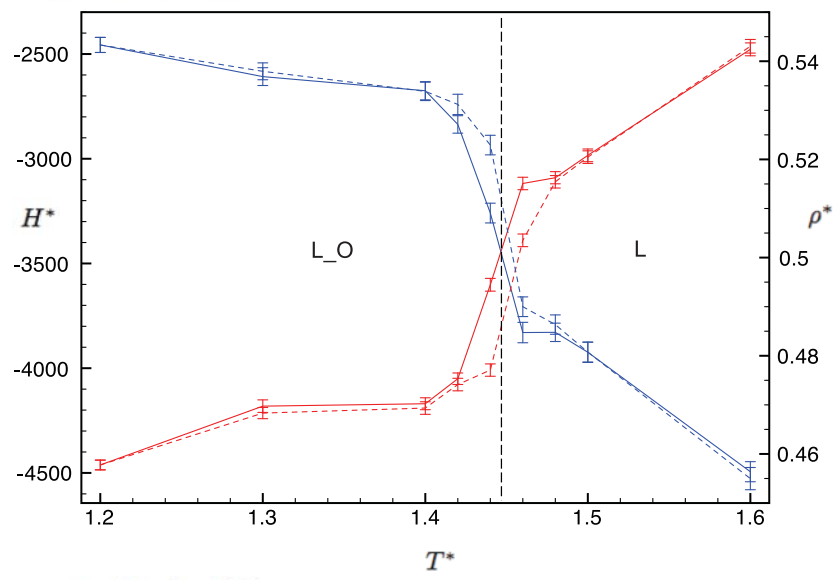

(b) $\quad \mu=5.0 \quad \lambda=0.25$

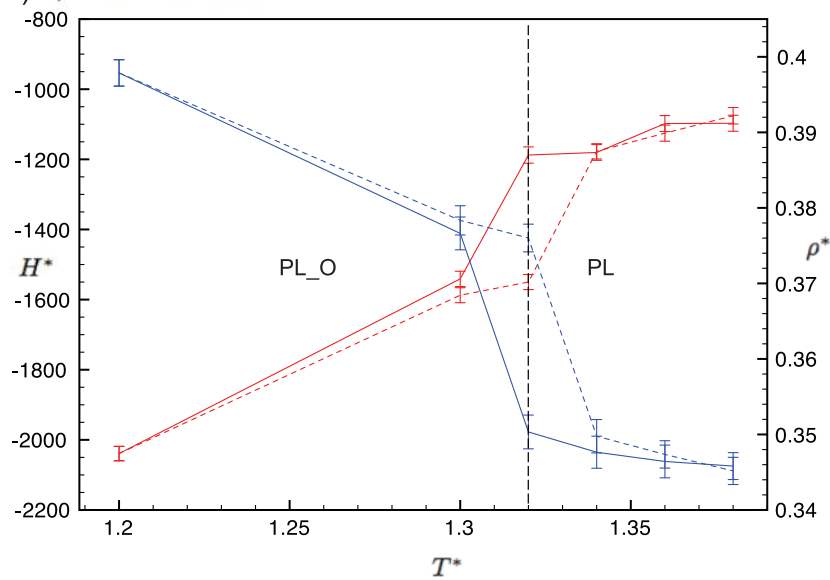

(c) $\quad \mu=6.0 \quad \lambda=0.1$

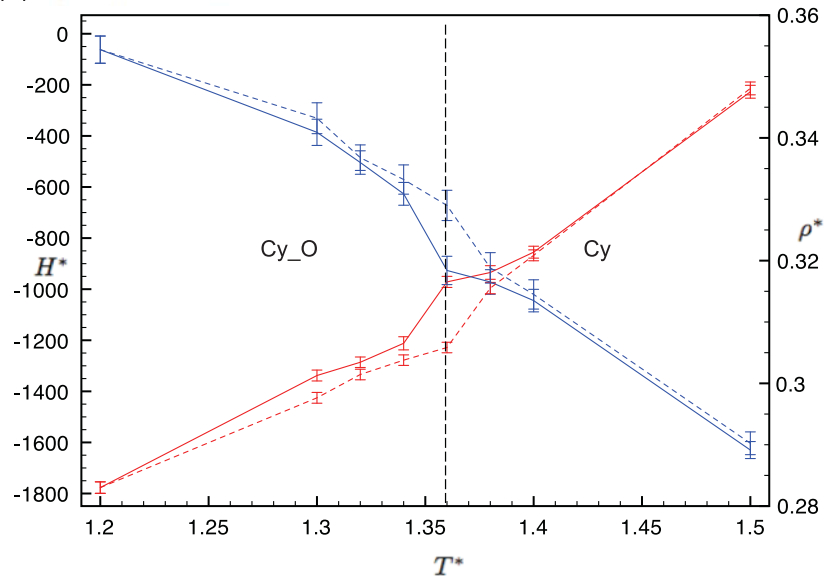

FIG. 4. $H^{*}$ and $\rho^{*}$ of the system as a function of $T^{*}$ for different values of $\mu$ and $\lambda$ at points near ordering phase transitions. Red line represents $H^{*}$ and blue line represents $\rho^{*}$. Dashed red line represents $H^{*}$ and dashed blue line represents $\rho^{*}$ for melting experiment by setting initial configuration as ordered phases. (a) $\mu=4.0, \lambda=1.0$; (b) $\mu=5.0, \lambda=0.25$; (c) $\mu=6.0$, $\lambda=0.1$. The dashed vertical lines represent the transition points. Due to very weak hysteresis of $H^{*}$ and $\rho^{*}$ curves, we can accurately determine the transition points.

to minimize the total free energy; therefore, the system forms a stable PL phase. ${ }^{16}$ By increasing the $\mu$ value, the disordered $\mathrm{D}$ region decreases and the region of L and PL phases increases. Also, the system crystallizes (experience phase transition to ordered phase) at a higher $T^{*}$ as we increase the $\mu$ value due to the added enthalpic contribution of disks. One can clearly observe these features if one compares the two phase diagrams: $\mu=1.0$ and $\mu=2.0$. At $\mu=2.0$, the $\mathrm{L}$ phase transforms into the $\mathrm{L}_{-} \mathrm{O}$ phase at high $\lambda$ and $T<1.25$. If we increase $\mu$ to 3.0 , the regions of $\mathrm{L}$ and PL phases increase a little more and ordered phase boundaries start to cover the L and PL phases. In other words, the system experiences phase transitions from $\mathrm{L}$ and PL phases to $\mathrm{L} \_\mathrm{O}$ and PL_O phases, respectively, at higher $T^{*}$. A similar trend is observed for $\mu=4.0$ except that a new Cy phase appears between the PL and D phases at low $\lambda$. The Cy phase can be explained as follows: if one increases $\mu$ considerably, the overall enthalpy of stacking disks increases, but the entropic role of coils remains the same. As a result, phase separation occurs at higher $T^{*}$ (we can see this from the fact that the D phase region decreases with increasing $\mu$ ). Therefore, we can think that if $\mu$ is increased, $\lambda$ is effectively decreased at elevated $T^{*}$. In this respect, at $\mu=4.0$ with low $\lambda$, coils effectively need more space than in the PL phase to increase free energy. The enlarged holes eventually connected together, and the cylinder phase is stabilized. As a result, at high $\mu$ and low $\lambda$, the system experiences $\mathrm{D} \rightarrow \mathrm{Cy} \rightarrow$ PL phase transitions as we decrease $T^{*}$. Another way of stating the previous train of thought is that the final structure to be formed depends on the interplay between the enthalpy and entropy of the system. In the large $\mu$ regime, the lateral disk interactions are not as important because the dominant enthalpic interaction is given by the central monomers. On the other hand, the entropy of the coils is increased if the curvature of the structure is larger. Thus, in the low $\lambda$ and high $\mu$ regime, one expects a cylindrical phase instead of a lamellar one. At $\mu=5.0$, regions of PL and $\mathrm{Cy}$ phases increase and $\mathrm{Cy} \_\mathrm{O}$ phase appear which shows a high degree of parallel packing compared to other ordered phases. Similarly, at $\mu=6.0$, PL and Cy phase regions increase, as well as the ordered phase boundary. At this value of $\mu$, it is hard to find the D phase except if one considers the region with high $T^{*}$ and low $\lambda$. In summary, phase behavior of disk-coil molecules with additional attractive interaction between central monomers of disks follows several rules listed below.

1. As $\lambda$ decreases, entropic role of coil increases.

2. As $\mu$ increases, we can think that $\lambda$ effectively decreases.

3. As the entropic role of coil increases, $\mathrm{L} \rightarrow \mathrm{PL} \rightarrow \mathrm{Cy}$ transformations occurs. But $\mathrm{Cy}$ phase appears only above a certain $\mu$ value.

4. As $T^{*}$ decreases, ordered phase boundaries start to appear, and these boundaries move to higher $T^{*}$ as $\mu$ increases, as expected.

In the next subsection, we will analyze the various ordered phases in a more quantitative fashion.

\section{Parallel packing of ordered phases}

Columnar structures with parallel stacked disks are common in discotic liquid crystals and understanding how to control their ordering is important for many device applications. Due to the additional central monomer attractive force, 
(a) $\mu=1.0$

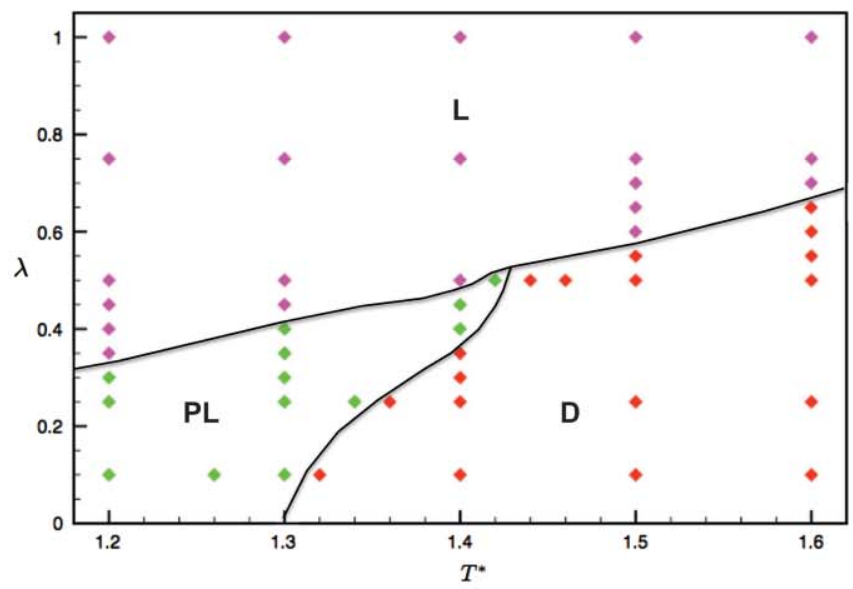

(c) $\mu=3.0$

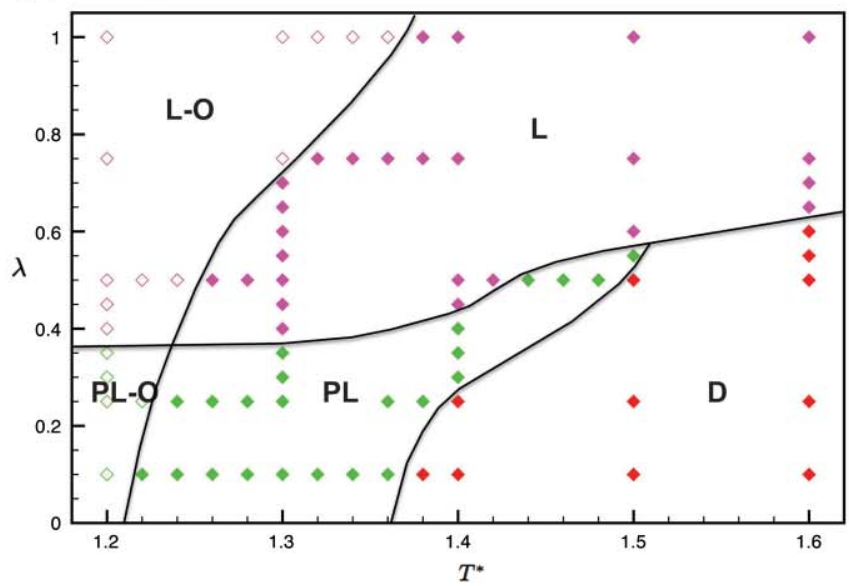

(e) $\mu=5.0$

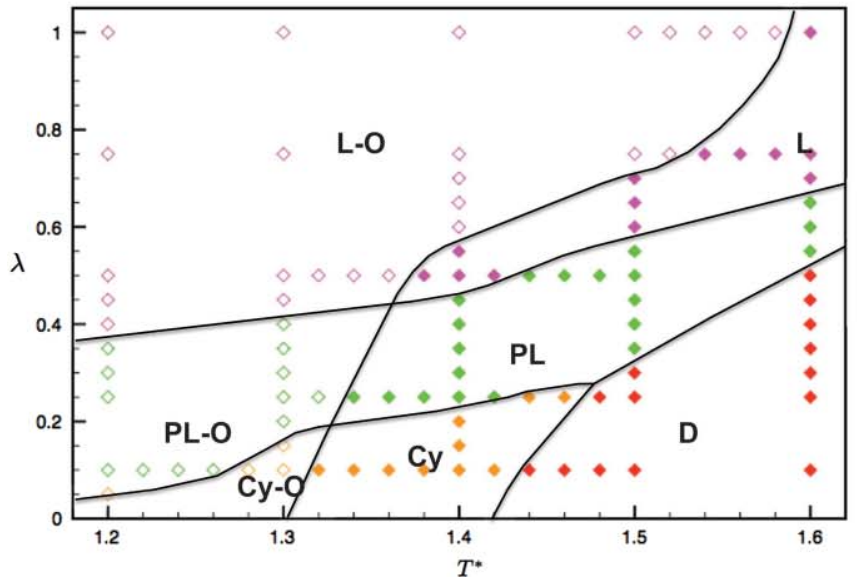

(b) $\mu=2.0$

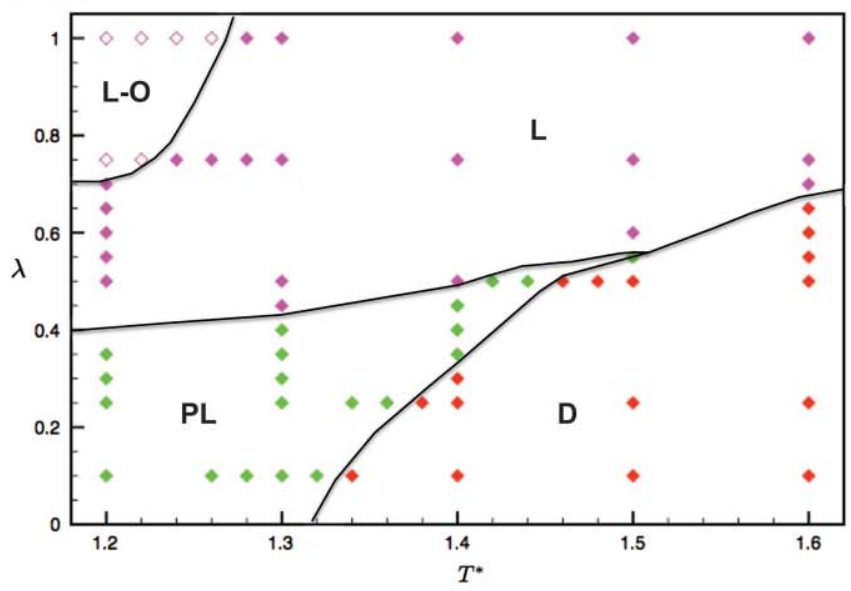

(d) $\mu=4.0$

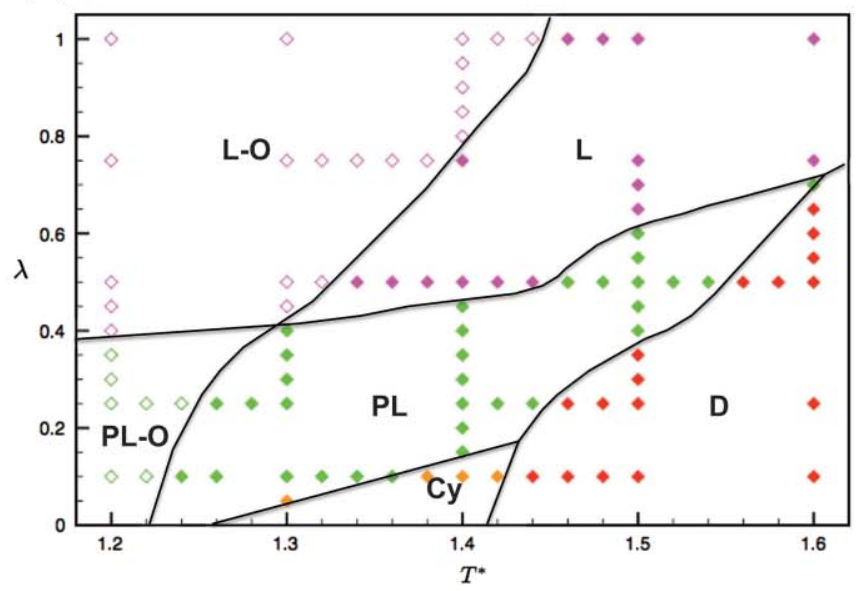

(f) $\mu=6.0$

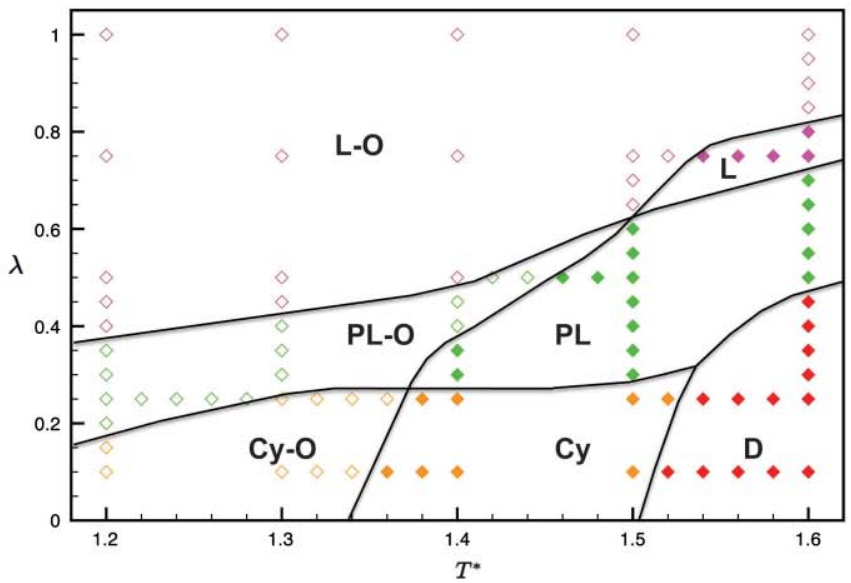

FIG. 5. Phase diagrams (in the $T^{*}$ vs $\lambda$ plane) of disk-coil molecules with different values of $\mu$ (a) $\mu=1.0$, (b) $\mu=2.0$, (c) $\mu=3.0$, (d) $\mu=4.0$, (e) $\mu=5.0$, and (f) $\mu=6.0$. Lines are guidelines for the eye only.

regional parallel stacking (4-6 disks are stacked in parallel) is obtained from L_O and PL_O phases, and long-ranged parallel stacking is reached at Cy_O phase as can be seen from Fig. 3. To study the parallel stacking of ordered phases, we define the integration of the radial distribution function as a correlation function to calculate the probability of parallel stacking of the disks. This function is defined in Eq. (2).

$$
P_{\text {parallel }}=\int_{0}^{1.5 r_{e q}^{*}} g\left(r^{*}\right) 4 \pi r^{* 2} d r^{*},
$$

where $g\left(r^{*}\right)$ is the radial distribution function of the central monomers of disks, and $r_{e q}^{*}=2^{1 / 6}$ in reduced equilibrium 


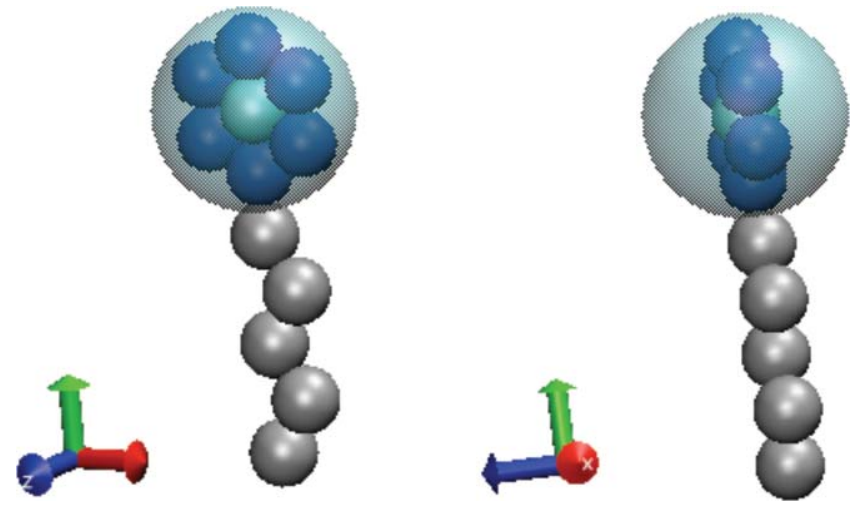

FIG. 6. Representation of disk-coil molecule with semi-transparent sphere of radius $1.5 r_{e q}^{*}$. $P_{\text {parallel }}$ represents the number of neighboring central monomers of disks within that sphere.

distance a unit of $\sigma$. When we integrate $g\left(r^{*}\right)$ with respect to certain volume, we can count how many central monomers exist within the integrated volume in average. Clearly, if we integrate over the simulation box, the result is $N-1=127$. Therefore, $P_{\text {parallel }}$ is a function that counts the number of central monomers which is equal to the number of disks within the sphere of radius $1.5 r_{e q}^{*}$. Figure 6 represents the sphere with radius $1.5 r_{e q}^{*}$ which has same center coordinate to the central monomer of disk. Here, $P_{\text {parallel }}$ counts the average number of neighboring disks whose central monomers stay within this sphere. We can clearly see that when molecules are perfectly parallel packed, $P_{\text {parallel }}$ reaches 2 , otherwise, $P_{\text {parallel }}$ cannot exceed 2.

The $P_{\text {parallel }}$ values of non-ordered phases $(\mathrm{D}, \mathrm{PL}, \mathrm{L}$, and Cy phases) are below 1.2. Because the attractive force between disks is not that strong for these phases, it is not likely to be packed parallel, therefore $P_{\text {parallel }}$ values are low. However, $P_{\text {parallel }}$ values of ordered phases (PL_O, L_O, and Cy_O phases) are mostly around $1.45 \pm 0.05$ because disks prefer to stack on top of each other due to the strong attractive force between central monomers. The only exception to this value is the Cy_O phase that displays a much higher $P_{\text {parallel }}=1.85 \pm 0.05$, that clearly shows that the disks are stacking in long trains in this phase. These values seem to hold under different conditions, such as $\mu=5$ and 6 . Based on $P_{\text {parallel }}$, we can also calculate the average number of stacked disks in a row from a rough calculation shown below.

$$
\begin{gathered}
P_{\text {parallel }}=\frac{2\left(N_{\text {parallel }}-2\right)+2}{N_{\text {parallel }}} \\
N_{\text {parallel }}=\frac{2}{2-P_{\text {parallel }}} .
\end{gathered}
$$

For PL_O and L_O phases, based on this calculation, average number of parallel stacked disks in a row is around 3.64. After we consider the presence of a defect, in average 4-6 disks are stacked in parallel without termination. These features are well represented in Figs. 3(a) and 3(b). The 4-6 parallel stacked disks are composed of a nanorod, and these nanorods are stacked to have lamellar or perforated lamellar morphology in any direction such as grains in typical materials. On the other hand, average number of stacked disks in parallel is around 13.3 for $\mathrm{Cy} \_\mathrm{O}$ phase. If we count some defects as you can see in Fig. 3(d), 14-16 disks should be stacked in parallel for one nanorod. From Fig. 3(d) super-aligned disks in parallel form long nanorods, and these nanorods of disks are stacked also in parallel to form cylinder which is similar to columnar structure. The difference of these phases that determine the average number of stacked disks in parallel is entropic coil of the molecule.

The coil portion of the molecule is very important to make disks stacked in parallel. To minimize surface energy, the director field vector (in our case, the vector perpendicular to the disk plane) should direct along the boundary of coil and disk regions. If the director field vector is perpendicular to that boundary, all seven monomers should be exposed toward the coil region, and this brings additional surface energy to the system. Director field vectors in L_O and PL_O phases are parallel to the coil rich region, but they still have two degrees of freedom within the lamellar plane. This implies that the director field vectors have two-dimensional degree of freedom, and the only way disks can be packed is in a hexagonal arrangement. This causes $60^{\circ}$ correlation of the director field vector. ${ }^{16}$ However, in Cy_O phase, entropic coils encapsulate the disk-rich region to form cylinders which provide essentially two-dimensional confinement. To minimize surface energy, all director field vectors must be aligned in one direction because they have only one degree of freedom. Therefore, with high $\mu$ and low $\lambda$, entropic coils enforce disks to be confined in a lower dimension, and this clearly enhances the probability of finding long parallel stacks of disks. This said, confinement due to the coils has a strong effect in the final arrangement of the disks.

\section{CONCLUSIONS}

In this article, we have investigated the phase behavior of disk-coil macromolecules with stacking interactions using Monte Carlo simulations. We found several phases depending on the temperature $T^{*}$, the relative strength of disk and coil portions $\lambda$, and the magnitude of the aforementioned stacking forces characterized by the parameter $\mu$. In addition to $\mathrm{D}$, $\mathrm{L}, \mathrm{PL}$, and $\mathrm{C}$ phases found in the simplest case of disk-coil molecules, ${ }^{16}$ we found L_O, PL_O, Cy, and Cy_O at elevated values of $\mu$. Disk rich layers of the ordered phases ( $\mathrm{L}_{-} \mathrm{O}$, PL_O, and Cy_O) are composed of parallel stacks of disks with a varying degree of alignment. We have constructed the phase diagrams as function of $\lambda$ and $T^{*}$ with six different $\mu$ values. At low $\mu$ and intermediate $T^{*}, \mathrm{~L} \rightarrow$ PL transition occurs with decreasing $\lambda$. At high $\mu$ and intermediate $T^{*}, \mathrm{~L} \rightarrow$ $\mathrm{PL} \rightarrow \mathrm{Cy}$ transitions occur when we decrease $\lambda$. We could understand these phase transitions by looking at the competition between entropic and enthalpic contributions of the coils. Ordered phase boundaries move to higher $T^{*}$ when increasing the stacking parameter $\mu$ because the enthalpic contribution of the disks increases, as expected.

To study the quality of the parallel stacking in the ordered phases, we introduced $P_{\text {parallel }}$ which is the average number of neighboring disks within short range distance. Based on this, we could calculate $N_{\text {parallel }}$, the average number of stacked disks in parallel in a row. For L_O and PL_O phases, 
only 4-6 disks are stacked in parallel in one nanorod. However, 14-16 disks are stacked in parallel in one nanorod in the Cy_O phase which provide very high degree of alignment. The difference between these three phases is only due to the entropic role of the coils. This high probability of parallel stacking is desired in the assembly of discotic liquid crystals, and our results may provide new design strategies for planar molecules that could be used in optoelectronic devices.

\section{ACKNOWLEDGMENTS}

This work was financially supported by a MITEI seed grant and an STX fellowship.

${ }^{1}$ D. Adam, P. Schuhmacher, J. Simmerer, L. Haussling, K. Siemensmeyer, K. H. Etzbach, H. Ringsdorf, and D. Haarer, Nature 371, 141 (1994).

${ }^{2}$ N. Boden, R. J. Bushby, J. Clements, and B. Movaghar, J. Mater. Chem. 9, 2081 (1999).

${ }^{3}$ L. Schmidt-Mende, A. Fechtenkotter, K. Mullen, E. Moons, R. H. Friend, and J. D. MacKenzie, Science 293, 1119 (2001).

${ }^{4}$ A. M. van de Craats, J. M. Warman, A. Fechtenkotter, J. D. Brand, M. A. Harbison, and K. Mullen, Adv. Mater. 11, 1469 (1999).

${ }^{5}$ S. A. Ponomarenko, N. I. Boiko, V. P. Shibaev, R. M. Richardson, I. J. Whitehouse, E. A. Rebrov, and A. M. Muzafarov, Macromolecules 33, 5549 (2000).

${ }^{6}$ M. Marcos, R. Martin-Rapun, A. Omenat, J. Barbera, and J. L. Serrano, Chem. Mater. 18, 1206 (2006)
${ }^{7}$ R. Mezzenga, J. Ruokolainen, N. Canilho, E. Kasemi, D. A. Schluter, W. B. Lee, and G. H. Fredrickson, Soft Matter 5, 92 (2009).

${ }^{8}$ J. S. Wu, W. Pisula, and K. Mullen, Chem. Rev. 107, 718 (2007).

${ }^{9}$ X. Feng, V. Marcon, W. Pisula, M. R. Hansen, J. Kirkpatrick, F. Grozema, D. Andrienko, K. Kremer, and K. Mullen, Nature Mater. 8, 412 (2009).

${ }^{10}$ D. Wu, L. Zhi, G. J. Bodwell, G. Gui, N. Tsao, and K. Mullen, Angew. Chem. Int. Ed. 46, 5417 (2007).

${ }^{11}$ D. Wu, W. Pisula, V. Enkelmann, X. Feng, and K. Mullen, J. Am. Chem. Soc. 131, 9620 (2009).

${ }^{12}$ J. Psencik, T. P. Ikonen, P. Laurinmaki, M. C. Merckel, S. J. Butcher, R. E. Serimaa, and R. Tuma, Biophys. J. 87, 1165 (2004).

${ }^{13}$ J. Psencik, J. B. Arellano, T. P. Ikonen, C. M. Borrego, P. Laurinmaki, S. J. Butcher, R. E. Serimaa, and R. Tuma, Biophys. J. 91, 1433 (2006).

${ }^{14}$ G. T. Oostergetel, M. Reus, A. G.M. Chew, D. A. Bryant, E. J. Boekema, and A. R. Holzwarth, FEBS Lett. 581, 5435 (2007).

${ }^{15}$ L. B. Modesto-Lopez, E. J. Thimsen, A. M. Collins, R. E. Blankenship, and P. Biswas, Energy Environ. Sci. 3, 216 (2010).

${ }^{16}$ Y. Kim and A. Alexander-Katz, J. Chem. Phys. 132, 174901 (2010).

${ }^{17}$ M. A. Horsch, Z. Zhang, and S. C. Glotzer, Phys. Rev. Lett. 95, 056105 (2005).

${ }^{18}$ M. Parrinello and A. Rahman, J. Appl. Phys. 52, 7182 (1981).

${ }^{19}$ J. R. Ray and A. Rahman, J. Chem. Phys. 80, 4423 (1984).

${ }^{20}$ J. A. C. Veerman and D. Frenkel, Phy. Rev. A 45, 5632 (1992).

${ }^{21}$ J. G. Gay and B. J. Berne, J. Chem. Phys. 74, 3316 (1981).

${ }^{22}$ M. A. Bates and G. R. Luckhurst, J. Chem. Phys. 104, 6696 (1996).

${ }^{23}$ R. Berardi, S. Orlandi, and C. Zannoni, J. Chem. Soc., Faraday Trans. 93, 1493 (1997).

${ }^{24}$ J. D. Weeks, D. Chandler, and H. C. Andersen, J. Chem. Phys. 54, 5237 (1971). 